\title{
Design and Analysis of Robotic Rover with Gripper Arm using Embedded C
}

\author{
Harmeet Singh, Sangeeta \\ Department of Computer Science \\ Punjab College of Technical Education (PCTE) \\ Baddowal, Punjab, India
}

\author{
Harpreet Kaur \\ Department of Electronics and Communication \\ Engineering \\ Sant Longowal Institute of Engineering and Technology \\ (SLIET) Longowal, Punjab, India
}

\begin{abstract}
This paper confers the development and working of robotic rover performing various autonomous tasks to identify, pick and drop an object at an appropriate position using microcontroller 8051 in conjunction with embedded C programming. The system employs infrared proximity sensors, DC geared motors, microcontroller board, etc. Robotic rover technology offers many applications in space explorations, military operations, etc. The motive of this research work is to design a wireless robotic rover being autonomous controlled that is capable of completing tasks with proliferated accuracy in smooth terrain.
\end{abstract}

Keywords: Robotic Rover; Embedded C; Microcontroller; Programming; Sensor

\section{INTRODUCTION}

Robotics technology is emanating at an expeditious pace, contributing new possibilities for automating tasks in many demanding applications, peculiarly in space explorations, military operations, underwater missions, etc. Especially, in space exploration, robotic devices are known as planetary rovers or simply rovers who aim at administering physical analysis of planetary terrains and astronomical bodies. The supervision of data about air pressure, climate, temperature, and other atmospheric aspects can be done by this advanced technology $[1,5,9]$. It is a space exploration vehicle designed to move across the surface of a planet or other heavenly body. Primarily, rovers can be self-governing or can be remotely controlled from the ground stations called as Remote Collaboration Center having very definite scientific objectives. Some rovers have been devised in the transportation of members of a human spaceflight crew; others have been partially or fully autonomous robots. Rovers or Unmanned Ground Vehicles (UGV) usually arrive at the planetary surface on a lander-style spacecraft $[2,7-8]$. The investigation of territories at the microscopic level, investigating the biological aspects of planetary surfaces, analyzing the composition of minerals, rocks, and soils, searching for liquid water in minerals, and measuring the ambient temperature, air pressure, and amount of dust in the landing site are some of its imperative appositeness [3]. Therefore, rovers are eminently computing systems that use complicated embedded software and algorithms to handle computational and processing functionalities.

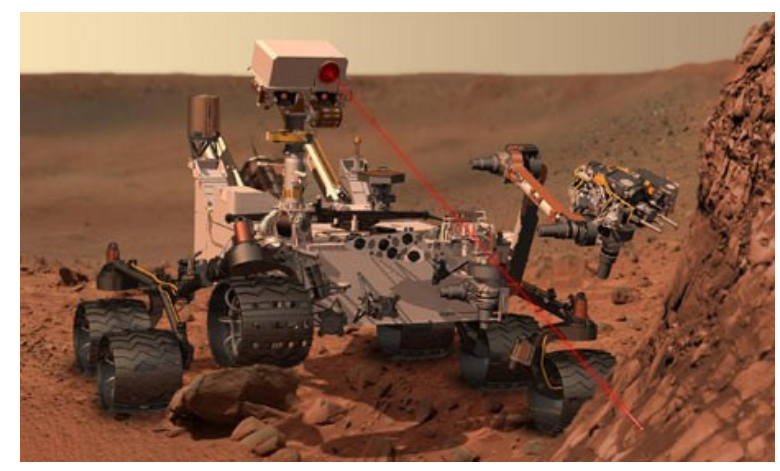

Figure.1 Schematic of Curiosity Rover on Mars
An autonomous robot has the competence to:

- Collect information about the environment, such as building maps of building interiors.

- Detect objects of interest such as people and vehicles.

- Travel between targets without human navigation and intervention.

- Disarm, or remove explosives.

- Repair itself without outside assistance.

The ongoing Mars exploration focuses around surface exploration using satellites and small, autonomous landexploration rovers which are used in predilection to manned missions as the cost is substantially lower and the rovers are more suited to the harsh planetary environment. Manned missions are still regarded as the 'holy grail' of topographical exploration; a current rover mission is aiming to scrutinize Mars with a pair of rovers, providing scope for coordination which was hitherto only possible with human exploration $[1,3-4,6]$. This paper is intended to study and discuss the design and development of robotic rover, its fully autonomous functionalities with various other capabilities.

\section{ROBOTIC ROVER DESIGN}

The structural part involves use of frames, beams, linkages, axles, etc. The mechanical parts/accessories comprise various types of gears (spurs, crowns, bevels, worms and differential gear systems), pulleys and belts, drive systems (differentials, castors, wheels and steering), etc. Pneumatics plays a vital role in generating specific pushing and pulling movements such as those simulating arms or leg movements. Pneumatic grippers are also used with advantage in robotics because of their simplicity and cost-effectiveness. The electrical items include DC and stepper motors, actuators, electrical grips, clutches and their control. The electronics part involves remote control, sensors (touch sensor, light sensor, collision sensor, etc), their interface circuitry and a microcontroller for overall control function.

The main objectives of the robotic rover project are given below: 
- It automatically senses the object of interest using infrared sensor.

- It picks up the object.

- It places the object at proper position.

- It moves forward in search of another object

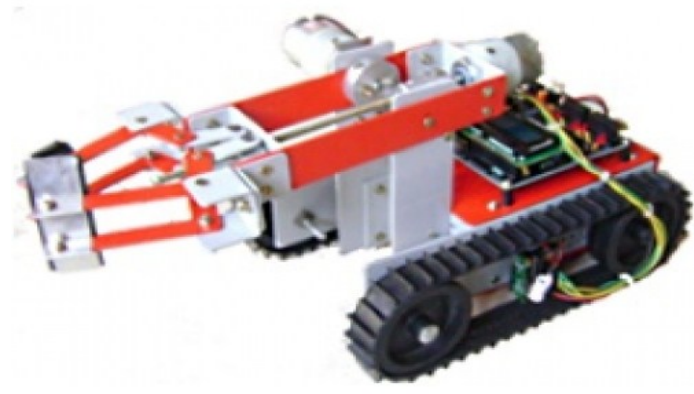

Figure.2 Robotic rover with gripper arm

\subsection{Hardware Design of Proposed Rover}

In this research work, an elementary robotic land rover that can be controlled remotely using primarily radio frequency (RF) module is presented. The tasks are autonomously controlled by the rover itself which is pre-programmed using embedded $\mathrm{C}$ programming. The RF remote control has the advantage of adequate range (up to 200 metres with proper antennae) besides being omnidirectional. On the other hand, an IR remote would function over a limited range of about a few metres.

The proposed land rover as shown in Figure 2 can move in forward and reverse directions with the gripper arm capable of advancing in left and right directions. While being deviated to left or right, the blinking of the corresponding LEDs takes place to indicate the direction of its turning. Similarly, during reverse movement, reversing LEDs would be lit. Front and rear bumpers are provided using long operating lever of micro switches to switch off the drive motors during any collision.

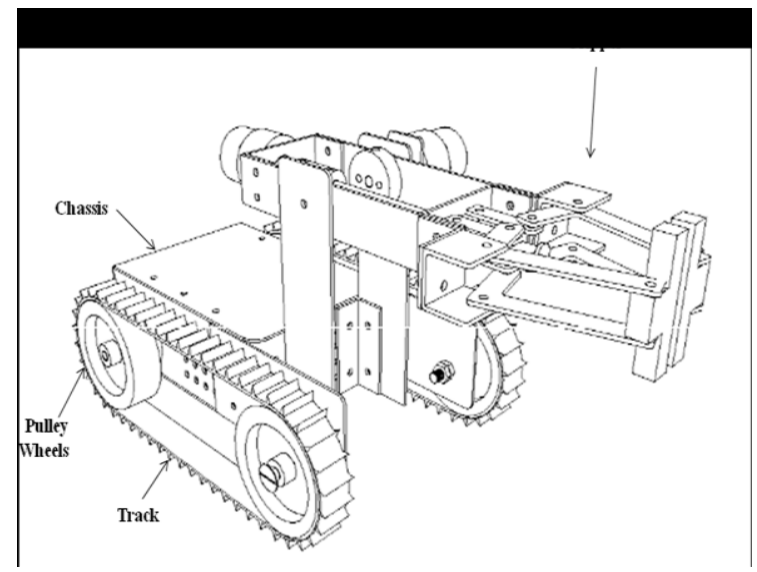

Figure. 3 Schematic diagram of robotic rover

The basic hardware of robotic rover as shown in Figure 3 consists of following parts:

- Aluminum Chassis
- Microcontroller board

- Infrared Proximity Sensor

- $\quad$ RF sensor

- $\quad$ Plastic wheels

- $\quad$ Four DC geared

- Gripper arm

The influential part of the robotic rover design is the gripper arm capable of picking up an object and doing functions of opening and closing the arm. Figure 4 delineates gripper arm consisting of various components. The individual parts were assembled to combine them into a single functional unit. The procedure of agglutination of components is also described in this section as described in Figure 5.

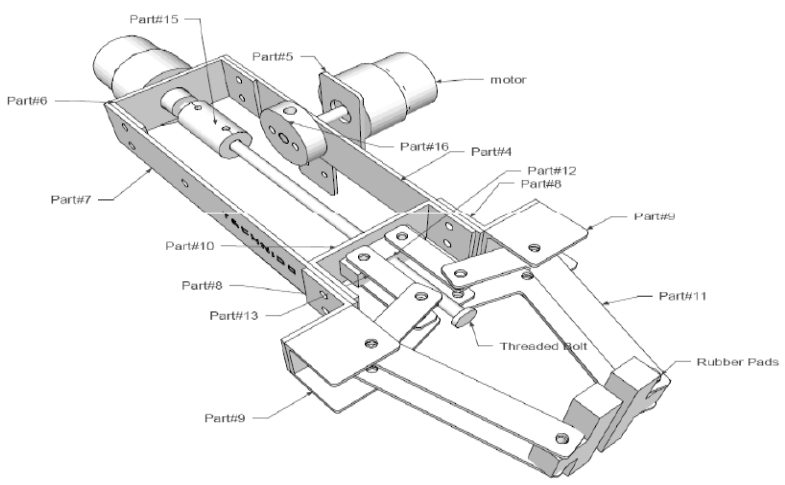

Figure. 4 Schematic diagram of gripper arm (reference: technido.com)
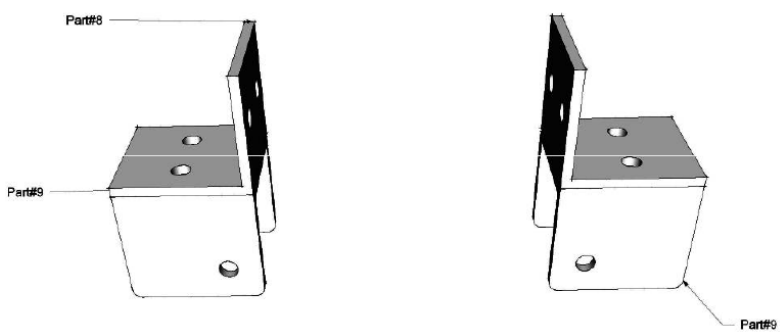

Figure. 5(a) Schematic diagram of gripper arm placing part\#8 over part\#9 and fixed using screw and nuts
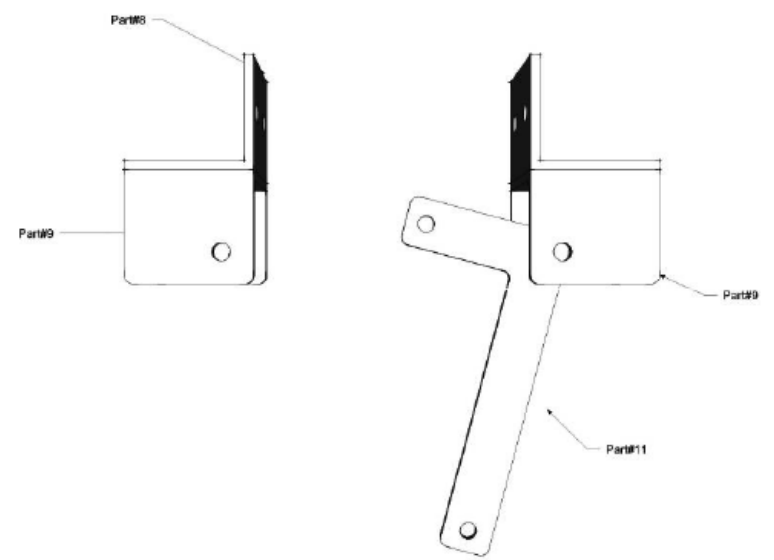

Figure. 5(b) Schematic diagram of gripper arm adding part\#11 to previous assembly 
International Journal of Computer Applications Technology and Research

Volume 4- Issue 2, 149 - 152, 2015, ISSN:- 2319-8656
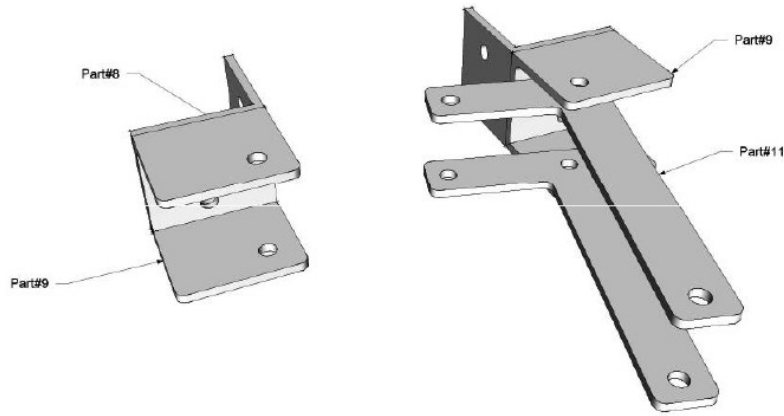

Figure. 5(c) Schematic diagram of gripper arm placing two L shaped part\#11 opposite to each other one above and one below
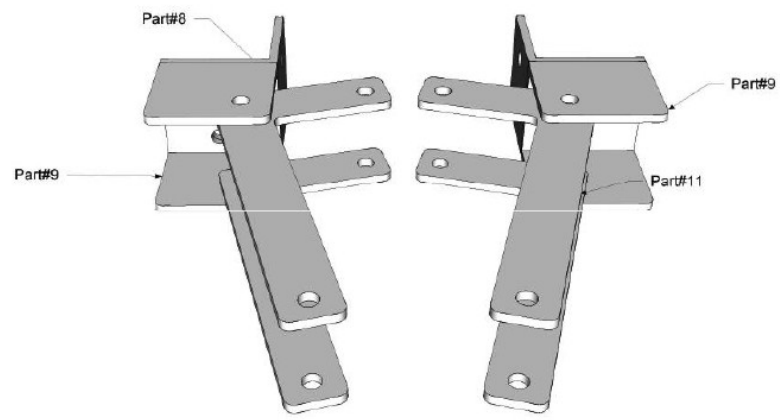

Figure. 5(d) Schematic diagram of gripper arm placing four L shaped part\#11 opposite to each other one above and one below

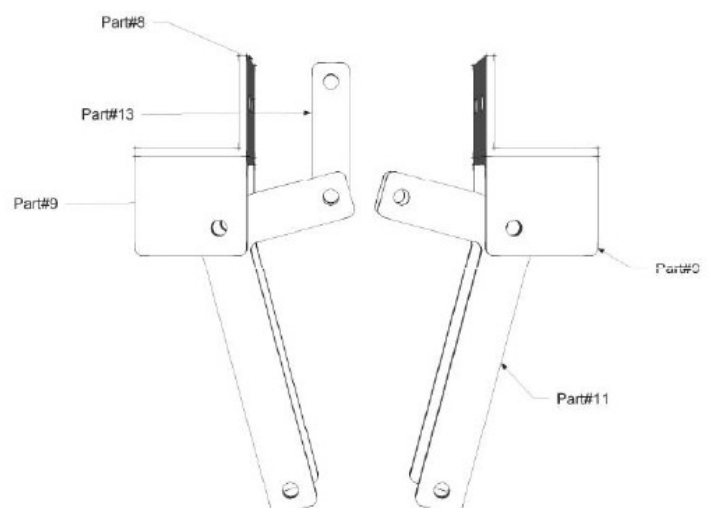

Figure. 5(e) Schematic diagram of gripper arm placing part\#13 loosely with previously fixed part\#11

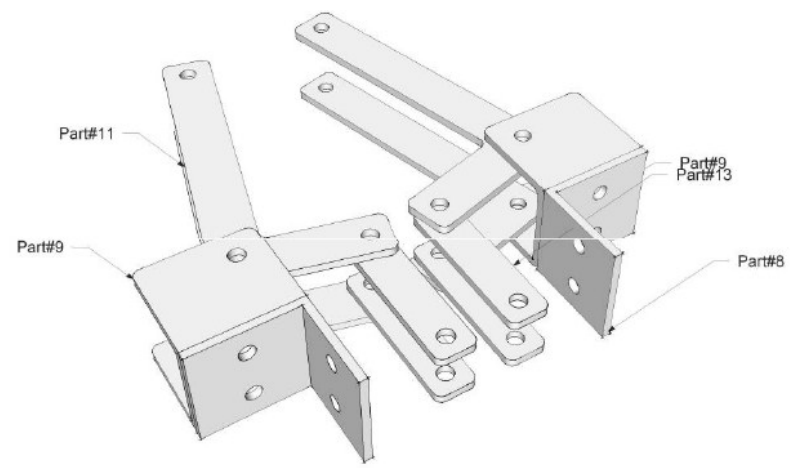

Figure. 5(f) Schematic diagram of gripper arm placing four part\#13 loosely with previously fixed part\#11

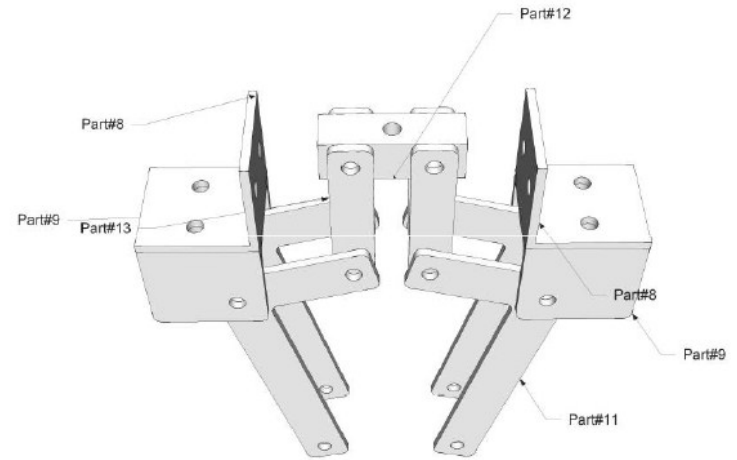

Figure. 5(g) Schematic diagram of gripper arm adding part\#12 made up of Aluminium holding all part\#13 in place

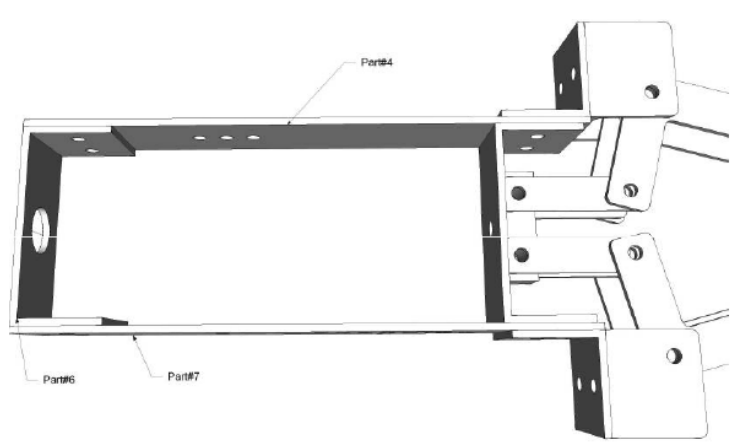

Figure. 5(h) Schematic diagram of gripper arm locating part\#6 in box and fixing it with part\#4 and part\#7

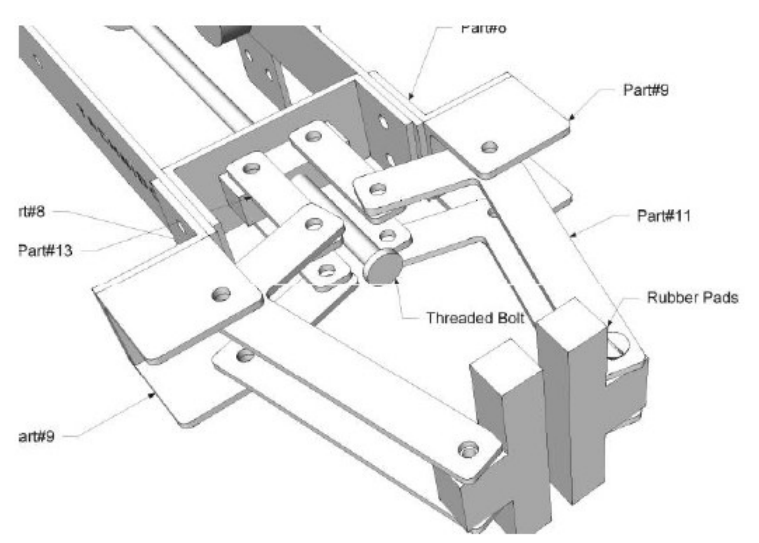

Figure. 5(i) Schematic diagram of gripper arm with threaded bolt and rubber pads

\subsection{Embedded C Programming}

The robotic rover programming was accomplished using microcontroller 8051 from NXP (founded by Philips) model P89V51RD2 which is a $40 \mathrm{MHz}, 5$ Volt microcontroller with 32 I/O lines, 3 Timers/Counters, 9 Interrupts/4 priority levels, $64 \mathrm{~K}+8 \mathrm{~K}$ FLASH, 1K on-chip RAM, SPI, Dual Data Pointers, WDT, 5-channel PCA. Keil $\mu$ Vision software was availed which combines project management, making facilities, source code editing, program debugging, and complete simulation in one powerful environment. The $\mu$ Vision development platform is easy-to-use and helps in quickly creating embedded programs. The $\mu$ Vision editor and 
debugger are assimilated in a single application that provides a seamless embedded project development environment. Flash magic software was used as program burner which is a PC tool for programming flash based microcontrollers from NXP using a serial or Ethernet protocol while in the target hardware.

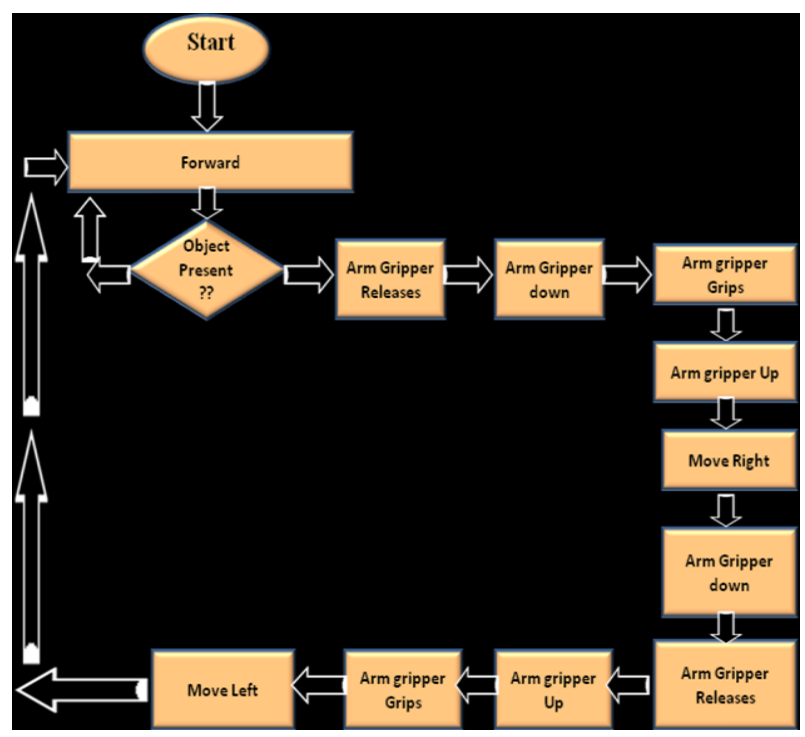

Figure. 6 Flow chart depicting working of proposed robotic rover

\subsection{Working of Robotic Rover}

Figure 6 illustrates the dynamics of robotic rover with gripper arm facility. This robot firstly moves in forward direction in search of an object. Here, the IR sensor plays a crucial role. The object is detected when the distance between the IR sensor and the object of interest is approximately $12 \mathrm{~cm}$ (the range can be further increased). If the object is encountered, its arm grip is released and moved in downward direction. Then the arm grip grasps again and picks up the object. It is placed in right direction where the arm gripper releases the object. It maneuvers in upward direction and robotic rover proceeds in forward direction. The programming of the whole operation is done in embedded $\mathrm{C}$ language using Keil $\mu$ Vision 3 tool from ARM Ltd.

\section{RESULTS}

Table 1. Operation versus time taken to complete different tasks"

\begin{tabular}{|c|c|c|}
\hline Steps & Operations & $\begin{array}{c}\text { Time taken to } \\
\text { complete (sec) }\end{array}$ \\
\hline Step 1 & Release of Gripper arm & 15 \\
\hline Step 2 & Down & 4 \\
\hline Step 3 & Grip (object) & 11 \\
\hline Step 4 & Up & 5 \\
\hline Step 5 & Right/left & 5 \\
\hline Step 6 & Down & 3 \\
\hline Step 7 & Release & 12 \\
\hline Step 8 & Up & 5 \\
\hline Step 9 & Grip (empty) & 15 \\
\hline Step 10 & Left/right & 5 \\
\hline
\end{tabular}

The robotic rover functionality comprises of some basic steps which were made fully autonomous using embedded $\mathrm{C}$ programming. The RF module was used to switch on/off the rover. Firstly, the release of empty gripper arm has taken a considerable time of $15 \mathrm{sec}$ followed by down movement of 4 sec. Due to presence of gravity of earth, down movement took minimum time among all other operations. Thenceforth, the object was grasped by the gripper arm covering a total of 11 sec. The gripping and releasing operations done by gripper arm clutched substantial seconds as shown in table 1 . Hereafter, the up movement of gripper arm took place with 5 seconds and positioning the object left/right. The object was then placed by a down release of 3 seconds since both the law of gravity and the weight of object exerted a downward force. Placing the object at proper place was the most momentous task. It took a second highest time of $12 \mathrm{sec}$ with release operation of the arm. Consequently, the arm was moved in upward direction with gripping of the arm. These operations were continued until and unless an object of interest was discovered.

\section{CONCLUSIONS}

This paper has bestowed an overview of the proposed robotic rover which can be used for sensing and navigation. It can be made fully autonomous with in-built camera, high performance controller, ultrasound sensors for future work. These enhancements will foster its capability for long range navigation. Being a latest technology in India, it would be benefaction for our space and defense applications.

\section{ACKNOWLEDGMENTS}

The authors are indebted to Kits'n'Spares, New Delhi and Technido Indore, India for providing Robotic Rover parts and technical help for making this work successful.

\section{REFERENCES}

[1] Zerigui A., WU X., Deng Z., "A Survey of Rover Control Systems", International Journal of Computer Sciences and Engineering Systems, Vol. 1, No. 4, pp. 105-109, 2007.

[2] Siegwart R., Nourbakhsh I., Scaramuzza D., Introduction to Autonomous Mobile Robots, 2nd edition, The MIT Press, 2011.

[3] Young A., Lunar and Planetary Rovers: The Wheels of Apollo and the Quest for Mars, Springer, 2006.

[4] Svitak, Amy, Cost of NASA's Next Mars Rover Hits Nearly $\$ 2.5$ Billion, http://www.space.com/10762-nasamars-rover-overbudget.html, retrieved 2011-02-03.

[5] Wesley T. Huntress JR., Mikhail Ya Marov, Soviet Robots in the Solar System: Mission Technologies and Discoveries, Springer, 2011.

[6] E. Colon, H. Sahli, and Y. Baudoin, -CoRoBa, a multi mobile robot control and simulation framework, International Journal of Advanced Robotic Systems, Vol. 3, No. 1, pp. 073-078, 2006.

[7] iRobot, Mobility Integration Software User's Guide, 2002.

[8] Cherry S., Robots, IEEE Spectrum, 2007.

[9] B. Gerkey, R. Vaughan, K. Sty, A. Howard, G. Sukhatme, and M. Mataric, - Most valuable player: A robot device server for distributed controlll, In Proceedings of the IEEE/RSJ International Conference on Intelligent Robots and Systems (IROS), 2001. 